\title{
A Survey on Geometric Dynamics of 4-Walker Manifold
}

\author{
Mehmet Tekkoyun \\ Department of Mathematics, Pamukkale University, Denizli, Turkey \\ E-mail:tekkoyun@pau.edu.tr \\ Received February 8, 2011; revised April 2, 2011; accepted April 15, 2011
}

\begin{abstract}
A Walker n-manifold is a semi-Riemannian n-manifold, which admits a field of parallel null $r$-planes, with $r \leq$ $\frac{n}{2}$. It is well-known that semi-Riemannian geometry has an important tool to describe spacetime events.

Therefore, solutions of some structures about 4-Walker manifold can be used to explain spacetime singularities. Then, here we present complex and paracomplex analogues of Lagrangian and Hamiltonian mechanical systems on 4-Walker manifold. Finally, the geometrical-physical results related to complex (paracomplex) mechanical systems are also discussed.
\end{abstract}

Keywords: Walker Manifolds, Lagrangian and Hamiltonian Mechanics

\section{Introduction}

As is well known, modern differential geometry plays an important role to explain the dynamics of Lagrangians. So, if $Q$ is an $m$-dimensional configuration manifold and $L: T Q \rightarrow \boldsymbol{R}$ is a regular Lagrangian function, then it is well-known that there is a unique vector field $X$ on $T Q$ such that dynamics equation is given by

$$
i_{X} \Phi_{L}=d E_{L}
$$

where $\Phi_{L}$ indicates the symplectic form. The triple ( $T Q$, $\left.\Phi_{L}, X\right)$ is called a Lagrangian system on the tangent bundle $T Q$.

Also, modern differential geometry provides a good framework in which develop the dynamics of Hamiltonians. Therefore, if $Q$ is an $m$-dimensional configuration manifold and $H: T^{*} Q \rightarrow \boldsymbol{R}$ is a regular Hamiltonian energy function, then there is a unique vector field $X$ on $T^{*} Q$ such that dynamics equation is given by

$$
i_{X} \Phi=d H
$$

where $\Phi$ indicates the symplectic form. The triple $\left(T^{*} Q\right.$, $\Phi, X)$ is called a Hamiltonian system on the cotangent bundle $T^{*} Q$.

Nowadays, there are many studies about Lagrangian and Hamiltonian dynamics, mechanics, formalisms, systems and equations [1-5] and there in. There are real, complex, paracomplex and other analogues. As we know it is possible to produce different analogues in different spaces.

Let $M_{2 n}$ be a Riemannian manifold with a neutral metric, i.e. with a semi-Riemannian metric $g$ of signature $(n, n)$. By a Walker n-manifold, we mean a semi-Riemannian manifold which admits a field of parallel null $r$-planes, with $r \leq \frac{n}{2}$. The canonical forms of the metrics were investigated by Walker [6]. Special interest manifolds are Walker manifolds of even dimensions $(n=$ $2 m$ ) admitting a field of null planes of maximum dimen$\operatorname{sionality}(r=m)$. An application of such a 4-dimensional Walker metric is given in [7]. Since the observation of the existence of almost complex structure on Walker 4-manifolds in [8], the Walker 4-manifolds and the almost Hermitian structures on the four-manifolds have been intensively studied, e.g., [9-14], etc. In this study, we present complex (paracomplex) analogues of Lagrangian and Hamiltonian mechanical systems on 4-Walker manifold. In the end, some geometrical-physical results about the obtained complex (paracomplex) mechanical systems are also given.

Throughout this paper, all mathematical objects and mappings are assumed to be smooth, i.e. infinitely differentiable and Einstein convention of summarizing is adopted. Denote by $M_{4}$ a Walker manifold. Then $\mathcal{F}\left(M_{4}\right), \quad \chi\left(M_{4}\right)$ and $\Lambda^{1}\left(M_{4}\right)$ are the set of func- 
tions on $M_{4}$, the set of vector fields on $M_{4}$ and the set of 1-forms on $M_{4}$, respectively.

\section{Walker Manifold $M_{4}$}

\subsection{Walker Metric}

A neutral metric $g$ on a 4-manifold $M_{4}$ is said to be Walker metric if there exists a 2-dimensional null distribution $D$ on $M_{4}$, which is parallel with respect to $g$. From Walker theorem [6], there is a system of coordinates with respect to which $g$ takes the local canonical form

$$
g=\left(g_{i j}\right)=\left(\begin{array}{llll}
0 & 0 & 1 & 0 \\
0 & 0 & 0 & 1 \\
1 & 0 & a & c \\
0 & 1 & c & b
\end{array}\right),
$$

where $a, b, c$ are smooth functions of the coordinates $\left(x_{1}\right.$, $\left.x_{2}, x_{3}, x_{4}\right)$. The parallel null 2-plane $D$ is spanned locally by $\left\{\partial_{1}, \partial_{2}\right\}$, where $\partial_{i}$ are the abbreviated forms of $\frac{\partial}{\partial_{i}}$, $i=x_{1}, x_{2}, x_{3}, x_{4}$.

\subsection{Proper Almost Complex Structure $\varphi$}

Let $\varphi$ be a proper almost complex structure on a Walker manifold $M_{4}$, which satisfies

1)

$$
\varphi^{2}=-I
$$

2) $g(\varphi X, Y)=-g(X, \varphi Y)$ (Hermitian property),

$$
\varphi \partial_{1}=\partial_{2}, \varphi \partial_{2}=-\partial_{1}
$$

3)

$$
\left(\varphi \text { induces a positive } \frac{\pi}{2}-\text { rotation on } D\right)
$$

We easily see that the above three properties defines $\varphi$ uniquely, i.e.

$$
\begin{aligned}
& \text { 1) } \varphi \partial_{1}=\partial_{2} \text {, } \\
& \text { 2) } \varphi \partial_{2}=-\partial_{1} \text {, } \\
& \text { 3) } \varphi \partial_{3}=-c \partial_{1}+\frac{1}{2}(a-b) \partial_{2}+\partial_{4} \text {, } \\
& \text { 4) } \varphi \partial_{4}=\frac{1}{2}(a-b) \partial_{1}+c \partial_{2}-\partial_{3} \text {, }
\end{aligned}
$$

If we write as $\varphi \partial_{1}=\sum_{j=1}^{4} \varphi_{i}^{j} \partial_{j}$, then from (4) we can read off the nonzero components $\varphi_{i}^{j}$ as follows:

$$
\begin{aligned}
& \varphi_{1}^{2}=-\varphi_{2}^{1}=\varphi_{3}^{4}=-\varphi_{4}^{3}=1, \\
& \varphi_{4}^{2}=-\varphi_{3}^{1}=c, \\
& \varphi_{3}^{2}=\varphi_{4}^{1}=\frac{1}{2}(a-b),
\end{aligned}
$$

i.e., $\varphi$ has the local components

$$
\varphi=\left(\varphi_{i}^{j}\right)=\left(\begin{array}{cccc}
0 & -1 & -c & \frac{1}{2}(a-b) \\
1 & 0 & \frac{1}{2}(a-b) & c \\
0 & 0 & 0 & 1 \\
0 & 0 & -1 & 0
\end{array}\right)
$$

with respect to the natural frame $\left\{\partial_{1}, \partial_{2}, \partial_{3}, \partial_{4}\right\}$ [14].

Similarly, we define a unique proper almost complex structure $\varphi^{*}$ as follows:

3) $\varphi^{*}\left(d_{3}\right)=-c d_{1}+\frac{1}{2}(a-b) d_{2}+d_{4}$,

$$
\text { 4) } \varphi^{*}\left(d_{4}\right)=\frac{1}{2}(a-b) d_{1}+c d_{2}-d_{3} \text {, }
$$

where $d_{i}$ are the abbreviated forms of $d x_{i}, i=x_{1}, x_{2}, x_{3}, x_{4}$.

\section{Lagrangian Mechanical Systems}

Now, our purpose is to obtain complex Euler-Lagrange equations for relativistic, quantum and classical mechanics on 4-dimensional Walker manifold $M_{4}$.

Let $M_{4}$ be a Walker manifold and $\left\{x_{1}, x_{2}, x_{3}, x_{4}\right\}$ be its coordinate functions. Let the semispray be the vector field $X$ determined by

$$
X=X^{1} \partial_{1}+X^{2} \partial_{2}+X^{3} \partial_{3}+X^{4} \partial_{4},
$$

where $X^{1}=\dot{x}_{1}, X^{2}=\dot{x}_{2}, X^{3}=\dot{x}_{3}, X^{4}=\dot{x}_{4}$ and the dot indicates the derivative with respect to time $t$. By means of the proper almost complex structure $\varphi$ given by (4), the vector field is defined by

$$
\begin{aligned}
V_{\varphi}= & \varphi(X)=X^{1} \partial_{2}-X^{2} \partial_{1} \\
& +X^{3}\left(-c \partial_{1}+\frac{1}{2}(a-b) \partial_{2}+\partial_{4}\right) \\
& +X^{4}\left(\frac{1}{2}(a-b) \partial_{1}+c \partial_{2}-\partial_{3}\right)
\end{aligned}
$$

which is named Liouville vector field on the Walker manifold $M_{4}$. The maps given by $T, P: M_{4} \rightarrow \boldsymbol{R}$ such that $T=\frac{1}{2} m_{i}\left(\dot{x}_{1}^{2}+\dot{x}_{2}^{2}+\dot{x}_{3}^{2}+\dot{x}_{4}^{2}\right), \quad P=m_{i} g h$ are said to be the kinetic energy and the potential energy of the system, respectively. Here $m_{i}, g$ and $h$ stand for mass of a mechanical system having $m$ particles, the gravity acceleration and distance to the origin of a mechanical system on Walker manifold $M_{4}$, respectively. Then $L: M_{4} \rightarrow \boldsymbol{R}$ is a map that satisfies the conditions; 1) $L=T-P$ is a Lagrangian function, 2) the function determined by 
$E_{L}^{\varphi}=V_{\varphi}(L)-L$, is energy function.

The function $i_{\varphi}$ induced by $\varphi$ and denoted by

$$
i_{\varphi} \omega\left(X_{1}, X_{2}, \cdots, X_{r}\right)=\sum_{i=1}^{r} \omega\left(X_{1}, \cdots, \varphi X_{i}, \cdots, X_{r}\right),
$$

is called vertical derivation, where $\omega \in \wedge^{r} M_{4}$, $X_{i} \in \chi\left(M_{4}\right)$. The vertical differentiation $d_{\varphi}$ is given by

$$
d_{\varphi}=\left[i_{\varphi}, d\right]=i_{\varphi} d-d i_{\varphi},
$$

where $d$ is the usual exterior derivation. For the almost complex structure $\varphi$ given by (4), the form on Walker manifold $M_{4}$ is the closed 2-form determined by $\Phi_{L}^{\varphi}=-d d_{\varphi} L$ such that

$$
\begin{gathered}
d_{\varphi}: \mathcal{F}\left(M_{4}\right) \rightarrow \wedge^{1} M_{4}, \\
d_{\varphi}=\partial_{2} d_{1}-\partial_{1} d_{2}+\left(-c \partial_{1}+\frac{1}{2}(a-b) \partial_{2}+\partial_{4}\right) d_{3} \\
+\left(\frac{1}{2}(a-b) \partial_{1}+c \partial_{2}-\partial_{3}\right) d_{4} .
\end{gathered}
$$

Through a direct computation using (7), the closed 2form $\Phi_{L}^{\varphi}$ is seen to be as follows:

$$
\begin{aligned}
\Phi_{L}^{\varphi}= & -\partial_{1^{\prime} 1}^{2} L d_{1^{\prime}} \wedge d_{1}+\partial_{1^{\prime} 1}^{2} L d_{1^{\prime}} \wedge d_{2}-\partial_{1^{\prime}}\left(-c \partial_{1} L+\frac{1}{2}(a-b) \partial_{2} L+\partial_{4} L\right) d_{1^{\prime}} \wedge d_{3} \\
& -\partial_{1^{\prime}}\left(\frac{1}{2}(a-b) \partial_{1} L+c \partial_{2} L-\partial_{3} L\right) d_{1^{\prime}} \wedge d_{4}-\partial_{2^{\prime} 2}^{2} L d_{2^{\prime}} \wedge d_{1}+\partial_{2^{\prime} 1}^{2} L d_{2^{\prime}} \wedge d_{2} \\
& -\partial_{2^{\prime}}\left(-c \partial_{1} L+\frac{1}{2}(a-b) \partial_{2} L+\partial_{4} L\right) d_{2^{\prime}} \wedge d_{3}-\partial_{2^{\prime}}\left(\frac{1}{2}(a-b) \partial_{1} L+c \partial_{2} L-\partial_{3} L\right) d_{2^{\prime}} \wedge d_{4} \\
& -\partial_{3^{\prime} 2}^{2} L d_{3^{\prime}} \wedge d_{1}+\partial_{3^{\prime} 1}^{2} L d_{3^{\prime}} \wedge d_{2}-\partial_{3^{\prime}}\left(-c \partial_{1} L+\frac{1}{2}(a-b) \partial_{2} L+\partial_{4} L\right) d_{3^{\prime}} \wedge d_{3} \\
& -\partial_{3^{\prime}}\left(\frac{1}{2}(a-b) \partial_{1} L+c \partial_{2} L-\partial_{3} L\right) d_{3^{\prime}} \wedge d_{4}-\partial_{4^{\prime} 2}^{2} L d_{4^{\prime}} \wedge d_{1}+\partial_{4^{\prime} 1}^{2} L d_{4^{\prime}} \wedge d_{2} \\
& -\partial_{4^{\prime}}\left(-c \partial_{1} L+\frac{1}{2}(a-b) \partial_{2} L+\partial_{4} L\right) d_{4^{\prime}} \wedge d_{3}-\partial_{4^{\prime}}\left(\frac{1}{2}(a-b) \partial_{1} L+c \partial_{2} L-\partial_{3} L\right) d_{4^{\prime}} \wedge d_{4}
\end{aligned}
$$

Then the energy function $E_{L}^{\varphi}$. is found as follows:

$$
E_{L}^{\varphi}=X^{1} \partial_{2} L-X^{2} \partial_{1} L+X^{3}\left(-c \partial_{1} L+\frac{1}{2}(a-b) \partial_{2} L+\partial_{4} L\right)+X^{4}\left(\frac{1}{2}(a-b) \partial_{1}+c \partial_{2}-\partial_{3}\right)-L .
$$

Suppose that a curve

$$
\begin{aligned}
& \alpha: R M_{4} \\
& \text { using (7) and (9) then we find } \\
& \partial_{t}\left(\partial_{2} L\right)-\partial_{1} L=0, \partial_{t}\left(-c \partial_{1} L+\frac{1}{2}(a-b) \partial_{2} L+\partial_{2} L\right)-\partial_{3} L=0, \\
& \partial_{t}\left(\partial_{1} L\right)+\partial_{2} L=0, \partial_{t}\left(\frac{1}{2}(a-b) \partial_{1} L+c \partial_{2} L-\partial_{3} L\right)-\partial_{4} L=0,
\end{aligned}
$$

such that the equations calculated in (10) are named complex Euler-Lagrange equations constructed on Walker manifold $M_{4}$ if 2-form $\Phi_{L}^{\varphi}$ is symplectic structure. Thus the triple $\left(M_{4}, \Phi_{L}^{\phi}, X\right)$ is named a complex mechanical system on Walker manifold $M_{4}$.

Now we obtain some corollaries about the equations raised in (10) thinking Remark (p. 387) in [14] and Proposition 4 in [8] and Corollary 4 in [15]:

Corollary 1: In (10), if $c=0$ and $a=b$ we find the equations as follows:

$$
\begin{aligned}
& \partial_{t}\left(\partial_{2} L\right)-\partial_{1} L=0, \partial_{t}\left(\partial_{2} L\right)-\partial_{3} L=0, \\
& \partial_{t}\left(\partial_{1} L\right)+\partial_{2} L=0, \partial_{t}\left(\partial_{3} L\right)+\partial_{4} L=0 .
\end{aligned}
$$

be an integral curve of semispray $X$. According to (1), using (7) and (9) then we find the following equations:
By means of Theorem 2 and Theorem 3 (p. 387 and p. 388) in [14], we can derive the following corollaries:

Corollary 2: If $a$ and $b$ satisfy the following PDEs:

$$
a_{1}+b_{1}=0, a_{2}+b_{2}=0 \text {, }
$$

then the equations in (10) are

$$
\begin{aligned}
& \partial_{t}\left(\partial_{2} L\right)-\partial_{1} L=0, \partial_{t}\left(\frac{1}{2}(a-b) \partial_{2} L+\partial_{2} L\right)-\partial_{3} L=0, \\
& \partial_{t}\left(\partial_{1} L\right)+\partial_{2} L=0, \partial_{t}\left(\frac{1}{2}(a-b) \partial_{1} L-\partial_{3} L\right)-\partial_{4} L=0,
\end{aligned}
$$

and have a solution. 
Corollary 3: If the following PDEs hold:

$$
a_{1}-b_{1}-2 c_{2}=0, a_{2}-b_{2}+2 c_{1}=0,
$$

then the equations in (10) have a solution.

\section{Hamiltonian Mechanical Systems}

This section is devoted to present complex Hamiltonian equations and Hamiltonian mechanical systems for relativistic, quantum and classical mechanics constructed on Walker manifold $M_{4}$.

Assume that a Liouville form and a 1-form on Walker manifold $M_{4}$ are shown $\lambda$ and $\omega$, respectively.

Let us consider

$$
\omega=x_{1} d_{1}+x_{2} d_{2}+x_{3} d_{3}+x_{4} d_{4} .
$$

Using (5), we have

$$
\begin{aligned}
\lambda= & \varphi^{*}(\omega)=x_{1} d_{2}-x_{2} d_{1}+x_{3}\left(-c d_{1}+\frac{1}{2}(a-b) d_{2}+d_{4}\right) \\
& +x_{4}\left(\frac{1}{2}(a-b) d_{1}+c d_{2}-d_{3}\right) .
\end{aligned}
$$

Assume that the vector field $X$ determined by (6) is Hamiltonian vector field associated with Hamiltonian energy function $H$. Then we find

$$
\begin{aligned}
\Phi= & -d \lambda=2 d_{2} \wedge d_{1}+2 d_{4} \wedge d_{3}+c\left(d_{3} \wedge d_{1}+d_{2} \wedge d_{4}\right) \\
& +\frac{1}{2}(a-b)\left(d_{2} \wedge d_{3}+d_{1} \wedge d_{4}\right),
\end{aligned}
$$

and

$$
\begin{aligned}
i_{X} \Phi= & \Phi(X)=2 X^{2} d_{1}-2 X^{1} d_{2}+2 X^{4} d_{3}-2 X^{3} d_{4} \\
& +c\left(X^{3} d_{1}-X^{1} d_{3}+X^{2} d_{4}-X^{4} d_{2}\right) \\
& +\frac{1}{2}(a-b)\left(X^{2} d_{3}-X^{3} d_{2}+X^{1} d_{4}-X^{4} d_{1}\right) .
\end{aligned}
$$

Furthermore, the differential of Hamiltonian energy function is obtained by

$$
d H=\partial_{1} H d_{1}+\partial_{2} H d_{2}+\partial_{3} H d_{3}+\partial_{4} H d_{4} .
$$

With respect to (2), if (16) and (17) are equaled, the Hamiltonian vector field is found as follows:

$$
\begin{aligned}
X= & \theta\left[\left(-2 c \partial_{3} H+a \partial_{4} H-b \partial_{4} H-4 \partial_{2} H\right) \partial_{1}\right. \\
& +\left(2 c \partial_{4} H+a \partial_{3} H-b \partial_{3} H+4 \partial_{1} H\right) \partial_{2} \\
& +\left(2 c \partial_{1} H-a \partial_{2} H+b \partial_{2} H-4 \partial_{4} H\right) \partial_{3} \\
& \left.+\left(-2 c \partial_{2} H-a \partial_{1} H+b \partial_{1} H+4 \partial_{3} H\right) \partial_{4}\right],
\end{aligned}
$$

where $\theta=\frac{2}{16+4 c^{2}+(a-b)^{2}}$.

Suppose that a curve

$$
\beta: I \subset \boldsymbol{R} \rightarrow M_{4}
$$

be an integral curve of the Hamiltonian vector field $X$, i.e.,

$$
X(\beta(t))=\dot{\beta}, t \in I .
$$

In the local coordinates, taking

$$
\beta(t)=\left(x_{1}, x_{2}, x_{3}, x_{4}\right)
$$

we find

$$
\dot{\beta}(t)=\frac{d_{1}}{d t} \partial_{1}+\frac{d_{2}}{d t} \partial_{2}+\frac{d_{3}}{d t} \partial_{3}+\frac{d_{4}}{d t} \partial_{4} .
$$

Using (19), (18), (20), it holds

$$
\begin{aligned}
& \frac{d_{1}}{d t}=\theta\left(-2 c \partial_{3} H+a \partial_{4} H-b \partial_{4} H-4 \partial_{2} H\right), \\
& \frac{d_{2}}{d t}=\theta\left(2 c \partial_{4} H+a \partial_{3} H-b \partial_{3} H+4 \partial_{1} H\right), \\
& \frac{d_{3}}{d t}=\theta\left(2 c \partial_{1} H-a \partial_{2} H+b \partial_{2} H-4 \partial_{4} H\right), \\
& \frac{d_{4}}{d t}=\theta\left(-2 c \partial_{2} H-a \partial_{1} H+b \partial_{1} H+4 \partial_{3} H\right) .
\end{aligned}
$$

As is known that if $\Phi$ is a closed form on Walker manifold $M_{4}$, then $\Phi$ is also a symplectic structure on Walker manifold $M_{4}$ [14]. Hence, the equations introduced in (21) are named complex Hamiltonian equations on Walker manifold $M_{4}$ if $\Phi$ is a symplectic structure. Then the triple $\left(M_{4}, \Phi_{\varphi^{*}}, X\right)$ is named a complex Hamiltonian mechanical system on Walker manifold $M_{4}$.

We obtain the following corollary considering the equations found in (21) using Remark (p. 387) in [14] and Proposition 4 in [8] and Corollary 4 in [15].

Corollary 4: In (21), if $c=0$ and $a=b$ we find the equations as follows:

$$
\begin{aligned}
& \frac{d_{1}}{d t}=-\frac{1}{2} \partial_{2} H, \frac{d_{2}}{d t}=\frac{1}{2} \partial_{1} H, \\
& \frac{d_{3}}{d t}=-\frac{1}{2} \partial_{4} H, \frac{d_{4}}{d t}=\frac{1}{2} \partial_{3} H .
\end{aligned}
$$

Taking Theorem 2 and Theorem 3 (p. 387 and p. 388) in [14], we can derive some corollaries as follows:

Corollary 5: If $a$ and $b$ satisfy the following PDEs:

$$
a_{1}+b_{1}=0, a_{2}+b_{2}=0,
$$

then the equations in (21) are 


$$
\begin{aligned}
\frac{d_{1}}{d t} & =\frac{2}{16+(a-b)^{2}}\left(a \partial_{4} H-b \partial_{4} H-4 \partial_{2} H\right), \\
\frac{d_{2}}{d t} & =\frac{2}{16+(a-b)^{2}}\left(a \partial_{3} H-b \partial_{3} H+4 \partial_{1} H\right), \\
\frac{d_{3}}{d t} & =\frac{2}{16+(a-b)^{2}}\left(-a \partial_{2} H+b \partial_{2} H-4 \partial_{4} H\right), \\
\frac{d_{4}}{d t} & =\frac{2}{16+(a-b)^{2}}\left(-a \partial_{1} H+b \partial_{1} H+4 \partial_{3} H\right),
\end{aligned}
$$

and have a solution.

Corollary 6: If the following PDEs hold:

$$
a_{1}-b_{1}-2 c_{2}=0, a_{2}-b_{2}+2 c_{1}=0,
$$

then the equations in (21) have a solution.

\section{Paracomplex Lagrangian and Hamiltonian Mechanical Systems}

In this section, we produce a paracomplex analogues of Lagrangian and Hamiltonian mechanical systems on a Walker manifold $M_{4}$.

Let $\varphi$ be a proper almost paracomplex structure on a Walker manifold $M_{4}$, which satisfies

$$
\varphi^{2}=I
$$

2) $g(\varphi X, Y)=-g(X, \varphi Y)($ Hermitian property $)$,

$$
\varphi \partial_{1}=-\partial_{1}, \varphi \partial_{2}=\partial_{2}
$$

Associated to any Walker metric (3) we consider a proper almost para-Hermitian structure $\varphi$ defined by

$$
\begin{gathered}
\varphi \partial_{1}=-\partial_{1}, \\
\text { 2) } \varphi \partial_{2}=\partial_{2}, \\
\text { 3) } \varphi \partial_{3}=-a \partial_{1}+\partial_{3}, \\
\text { 4) } \varphi \partial_{4}=b \partial_{2}-\partial_{4},
\end{gathered}
$$

where $\left\{\partial_{i}\right\}, i=x_{1}, x_{2}, x_{3}, x_{4}$. denotes the coordinate bases [9].

If we write as $\varphi \partial_{i}=\sum_{j=1}^{4} \varphi_{i}^{j} \partial_{j}$, then from (24) we can read off the nonzero components $\varphi_{i}^{j}$ as follows:

$$
\varphi_{1}^{1}=-\varphi_{2}^{2}=-\varphi_{3}^{3}=-\varphi_{4}^{4}=-1, \varphi_{3}^{1}=-a, \varphi_{4}^{2}=b,
$$

i.e., $\varphi$ has the local components

$$
\varphi=\left(\varphi_{i}^{j}\right)=\left(\begin{array}{cccc}
-1 & 0 & -a & 0 \\
0 & 1 & 0 & b \\
0 & 0 & 1 & 0 \\
0 & 0 & 0 & -1
\end{array}\right)
$$

with respect to the natural frame $\left\{\partial_{1}, \partial_{2}, \partial_{3}, \partial_{4}\right\}$.

According to the proper almost paracomplex structure $\varphi$, the 2-form $\Phi_{L}^{\varphi}$ is given by as follows:

$$
\begin{aligned}
& \Phi_{L}^{\varphi}=\partial_{1^{\prime} 1}^{2} L d_{1^{\prime}} \wedge d_{1}-\partial_{1^{\prime} 2}^{2} L d_{1^{\prime}} \wedge d_{2} \\
& -\left(-a \partial_{1^{\prime} 1}^{2} L+\partial_{1^{\prime} 3}^{2} L\right) d_{1^{\prime}} \wedge d_{3}-\left(b \partial_{1^{\prime} 2}^{2} L-\partial_{1^{\prime} 4}^{2} L\right) d_{1^{\prime}} \wedge d_{4} \\
& +\partial_{2^{\prime} 1}^{2} L d_{2^{\prime}} \wedge d_{1}-\partial_{2^{\prime} 2}^{2} L d_{2^{\prime}} \wedge d_{2} \\
& -\left(-a \partial_{2^{\prime} 1}^{2} L+\partial_{2^{\prime} 3}^{2} L\right) d_{2^{\prime}} \wedge d_{3}-\left(b \partial_{2^{\prime} 2}^{2} L-\partial_{2^{\prime} 4}^{2} L\right) d_{2^{\prime}} \wedge d_{4} \\
& +\partial_{3^{\prime} 1}^{2} L d_{3^{\prime}} \wedge d_{1}-\partial_{3^{\prime} 2}^{2} L d_{3^{\prime}} \wedge d_{2} \\
& -\left(-a \partial_{3^{\prime} 1}^{2} L+\partial_{3^{\prime} 3}^{2} L\right) d_{3^{\prime}} \wedge d_{3}-\left(b \partial_{3^{\prime} 2}^{2} L-\partial_{3^{\prime} 4}^{2} L\right) d_{3^{\prime}} \wedge d_{4} \\
& +\partial_{4^{\prime} 1}^{2} L d_{4^{\prime}} \wedge d_{1}-\partial_{4^{\prime} 2}^{2} L d_{4^{\prime}} \wedge d_{2} \\
& -\left(-a \partial_{4^{\prime} 1}^{2} L+\partial_{4^{\prime} 3}^{2} L\right) d_{4^{\prime}} \wedge d_{3}-\left(b \partial_{4^{\prime} 2}^{2} L-\partial_{4^{\prime} 4}^{2} L\right) d_{4^{\prime}} \wedge d_{4} .
\end{aligned}
$$

By means of $\Phi_{L}^{\varphi}$, using similar method in Section 3, we get

$$
\begin{aligned}
& \frac{\partial}{\partial t}\left(\partial_{1} L\right)+\partial_{1} L=0, \frac{\partial}{\partial t}\left(\partial_{2} L\right)-\partial_{2} L=0, \\
& \frac{\partial}{\partial t}\left(-a \partial_{1} L+\partial_{3} L\right)-\partial_{3} L=0, \\
& \frac{\partial}{\partial t}\left(b \partial_{2} L-\partial_{4} L\right)-\partial_{4} L=0,
\end{aligned}
$$

such that the equations calculated in (25) are named a paracomplex Euler-Lagrange equations constructed on the Walker manifold $M_{4}$ if the 2 -form $\Phi_{L}^{\varphi}$ is symplectic structure. Hence the triple $\left(M_{4}, \Phi_{L}^{\varphi}, X\right)$ is named a paracomplex mechanical system on the Walker manifold $M_{4}$.

Now, let us consider the proper almost paracomplex structure $\varphi^{*}$ :

$$
\begin{gathered}
\text { 1) } \varphi^{*}\left(d_{1}\right)=-d_{1}, \\
\text { 2) } \varphi^{*}\left(d_{2}\right)=d_{2}, \\
\text { 3) } \varphi^{*}\left(d_{3}\right)=-a d_{1}+d_{3}, \\
\text { 4) } \varphi^{*}\left(d_{4}\right)=b d_{2}-d_{4} .
\end{gathered}
$$

For the proper almost paracomplex structure $\varphi^{*}$, the 2-form $\Phi_{\varphi^{*}}$ is given by

$$
\begin{aligned}
\Phi_{\varphi^{*}}= & -d \lambda=2 d_{2} \wedge d_{1}+2 d_{3} \wedge d_{4} \\
& +a d_{4} \wedge d_{1}+b d_{2} \wedge d_{3},
\end{aligned}
$$

By using similar method in section 4 , by means of $\Phi_{\varphi^{*}}$, we have

$$
\begin{aligned}
& \frac{d_{1}}{d t}=\frac{1}{4+a b}\left(-2 \partial_{2} H-b \partial_{4} H\right), \\
& \frac{d_{2}}{d t}=\frac{1}{4+a b}\left(2 \partial_{1} H+a \partial_{3} H\right), \\
& \frac{d_{3}}{d t}=\frac{1}{4+a b}\left(-a \partial_{2} H+2 \partial_{4} H\right), \\
& \frac{d_{4}}{d t}=\frac{1}{4+a b}\left(b \partial_{1} H-2 \partial_{3} H\right) .
\end{aligned}
$$


Hence, the equations introduced in (27) are named a paracomplex Hamiltonian equations on the Walker manifold $M_{4}$ if the 2-form $\Phi_{L}^{\varphi}$ is symplectic structure. Then the triple $\left(M_{4}, \Phi_{\varphi^{*}}, X\right)$ is named a paracomplex Hamiltonian mechanical system on the Walker manifold $M_{4}$.

\section{Discussions}

From above, complex and paracomplex Lagrangian mechanical systems have intrinsically been described on a Walker manifold $M_{4}$ : The paths of semispray $X$ on Walker manifold $M_{4}$ are the solutions of complex and paracomplex Euler-Lagrange equations raised in (10) and (25).

Also, complex and paracomplex Hamiltonian mechanical systems have intrinsically been described on a Walker manifold $M_{4}$. The paths of Hamilton vector field $X$ on the Walker manifold $M_{4}$ are the solutions of complex and paracomplex Hamiltonian equations raised in (21) and (27).

One easily see that the complex (paracomplex) EulerLagrange and Hamilton equations introduced in $[4,5]$ are equivalent those in (10) and (21) ((25) and (27)) if $\mathrm{a}=\mathrm{b}$ $=0$.

One can be proved that the here obtained equations are very important to explain the space-time mechanicalphysical problems. Therefore, the found equations are only considered to be a first step to realize how complex (paracomplex) structures on a Walker manifold has been used in solving problems in different physical areas.

For further research, the complex (paracomplex) Lagrangian and Hamiltonian mechanical equations derived here are suggested to deal with problems in electrical, magnetical and gravitational fields of relativistic, quantum and classical mechanics of physics.

\section{References}

[1] M. De Leon and P. R. Rodrigues, "Methods of Differential Geometry in Analytical Mechanics," North-Holland Mathematics Studies, Elsevier, Amsterdam, 1989.

[2] M. De Leon and P. R. Rodrigues, "Second-Order Differential Equations and Non-Conservative Lagrangian Mechanics," Journal of Physics A: Mathematical and General, Vol. 20, 1987, pp. 393-5396.

[3] M. Zambine, "Hamiltonian Perspective on Generalized Complex Structure," Communications in Mathematical
Physics, Vol. 263, 2006, pp. 711-722. doi:10.1007/s00220-005-1512-5

[4] M. Tekkoyun and M. Görgülü, "Higher Order Complex Lagrangian and Hamiltonian Mechanics Systems," Physical Letters A, Vol. 357, 2006, pp. 261-269. doi:10.1016/i.physleta.2006.04.049

[5] M. Tekkoyun, "On Para-Euler-Lagrange and Para-Hamiltonian Equations," Physical Letters A, Vol. 340, No. 1-4, 2005, pp. 7-11.

[6] A. G. Walker, "Canonical Form for a Rimannian Space with a Paralel Field of Null Planes," Quarterly of Applied Mathematics Oxford, Vol. 1, No. 2, 1950, pp. 69-79.

[7] R. Ghanam and G. Thompson, "The Holonomy Lie Algebras of Neutral Metrics in Dimension Four," Journal of Mathematical Physics, Vol. 42, 2001, pp. 2266-2284. doi:10.1063/1.1362284

[8] Y. Matsushita, "Four-Dimensional Walker Metrics and Symplectic Structures," Journal of Geometry and Physics, Vol. 52, 2004, pp. 89-99. doi:10.1016/j.geomphys.2004.02.009

[9] J. Carlos Diaz-Ramos, E. Garcia-Rio and R. VazquezLorenzo, "Osserman Metrics on Walker 4-Manifolds Equipped with a Para-Hermitian Structure," Matematica Contemporanea, Vol. 30, 2006, pp. 91-108.

[10] M. Chaichi, E. Garca-Ro and Y. Matsushita, "Curvature Properties of Four-Dimensional Walker Metrics," Classical and Quantum Gravity, Vol. 22, No. 3, 2005, pp. 559-577. doi:10.1088/0264-9381/22/3/008

[11] J. Davidov and O. Mukarov, "Self-DualWalker Metrics with Two-Step Nilpotent Ricci Operator," Journal of Geometry and Physics, Vol. 57, 2006, pp. 157-165. doi:10.1016/j.geomphys.2006.02.007

[12] J. C. Diaz-Ramos, E. Garca-Ro and R. Vazquez-Lorenzo, "New Examples of Osserman Metrics with Nondiagonalizable Jacobi Operators," Differential Geometry and Its Applications, Vol. 24, 2006, pp. 433-442. doi:10.1016/j.difgeo.2006.02.006

[13] E. Garca-Ro, Z. Raki and M. E. Vazquez-Abal, "FourDimensional Indefinite Kähler Osserman Manifolds," Journal of Mathematical Physics, Vol. 46, 2005, p. 073505 (11 Pages).

[14] Y. Matsushita, "Walker 4-Manifolds with Proper Almost Complex Structures," Journal of Geometry and Physics, Vol. 55, 2005, pp. 385-398. doi:10.1016/j.geomphys.2004.12.014

[15] E. Garca-Ro, S. Haze, N. Katayama and Y. Matsushita, "Symplectic, Hermitian and Kähler Structures on Walker 4-Manifolds," Journal of Geometry, Vol. 90, 2008, pp. 56-65. doi:10.1007/s00022-008-1999-y 